\title{
Analysis of small-signal stability of power systems with photovoltaic generators
}

\author{
Shinsaku Izumi ${ }^{1}$ (D) Yuya Karakawa ${ }^{2} \cdot$ Xin Xin $^{1}$
}

Received: 13 February 2019 / Accepted: 23 April 2019 / Published online: 14 May 2019

(c) The Author(s) 2019

\begin{abstract}
This paper presents a theoretical analysis of the small-signal stability of a power system in which a synchronous generator and a photovoltaic (PV) generator supply power to an infinite bus. The problem considered here is to investigate the existence of the equilibrium points of the system and their stability. In terms of this problem, by focusing on the condition to be satisfied by the equilibrium points and appropriately using the intermediate value theorem, we derive a sufficient condition on the magnitude of the PV current for the existence of the equilibrium points. The condition is given as inequalities with respect to the system parameters. These inequalities show that, if the power system from which the PV generator is removed has equilibrium points, then equilibrium points exist also in the original system as long as the PV current is small. Moreover, we analyze the stability of the equilibrium points and show that the equilibrium points found under our existence condition are asymptotically stable. These results imply that, when the PV current is below a certain level, the existence of the asymptotically stable equilibrium points is preserved even though the PV generator is introduced.
\end{abstract}

Keywords Power systems $\cdot$ Photovoltaic generators $\cdot$ Small-signal stability $\cdot$ Equilibrium points

\section{Introduction}

Modern power systems are characterized by a high penetration of photovoltaic (PV) generators [11]. This is because the increasing use of electricity generated in environmentally friendly ways, such as by using solar energy, is driven by critical concerns over the depletion of fossil fuels and greenhouse gas emissions. Since PV generators convert solar energy directly into electricity, there is no consumption of fossil fuels and hence are no greenhouse gas emissions.

However, the introduction of PV generators affects the performance of power systems. For example, PV penetration

This work was partly supported by JSPS KAKENHI Grant Number $16 \mathrm{~K} 18124$.

Shinsaku Izumi

izumi@cse.oka-pu.ac.jp

Xin Xin

xxin@cse.oka-pu.ac.jp

1 Faculty of Computer Science and Systems Engineering, Okayama Prefectural University, 111 Kuboki, Soja, Okayama 719-1197, Japan

2 West Japan Railway Company, 4-1-60, Noda, Kita-ku, Okayama 700-0971, Japan degrades the stability of power systems because PV generators do not have the synchronizing torque [6], i.e., internal torque to preserve the synchronization of generators. To solve this problem, many studies have been conducted to date. For instance, a model of a PV generator has been constructed to study the interaction of $\mathrm{PV}$ generators with power systems [12]. Other researchers have analyzed the impact of PV penetration on the transient stability $[10,14]$ and voltage characteristics $[1,7,13,16]$ of power systems. Additionally, controller design for PV systems connected to power grids has been carried out $[5,9,15]$.

Our interest in this research is to determine the impact of the penetration of PV generators on the small-signal stability [6] of power systems. This stability indicates the ability of power systems to maintain the synchronization against small disturbances such as variations in loads and power. The motivation for considering the small-signal stability is that this characteristic is fundamental to the problem-free operation of power systems. In fact, a power system without the smallsignal stability is destabilized even by small disturbances, which ultimately leads to the disruption of the power supply.

The purpose of this paper is to investigate the impact of PV penetration on the small-signal stability. For this purpose, we consider the system in Fig. 1. This is composed 


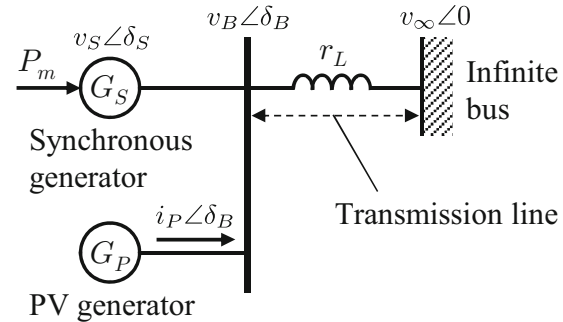

Fig. 1 Power system $\Sigma$ with PV generator

of a synchronous generator, a PV generator, and an infinite bus corresponding to a large system to which the two generators are connected. The generators supply power to the infinite bus through a transmission line. The system in Fig. 1 focuses only on the synchronous generator and the PV generator and thus is suitable for investigating the direct impact of $\mathrm{PV}$ penetration on the behavior of the synchronous generator. For this system, we consider the stability of the equilibrium points, i.e., the states in which the electrical output of the synchronous generator is in balance with the mechanical input to it, as the small-signal stability. We then address the problem of verifying the existence of the equilibrium points and analyzing their stability.

In terms of the above problem, this paper makes two contributions. First, we derive a sufficient condition on the magnitude of the PV current for the existence of the equilibrium points. As a result, we show that, if the power system from which the PV generator is removed has equilibrium points, then equilibrium points exist also in the original system as long as the PV current is small. The key idea behind this result is to employ the intermediate value theorem. A straightforward method for solving our problem would be to derive the equilibrium points and clarify their properties. However, deriving the equilibrium points is difficult because it is necessary to solve a nonlinear equation with trigonometric functions and their inverses from the presence of the PV generator. We address this difficulty by focusing on the condition to be satisfied by the equilibrium points and by appropriately using the intermediate value theorem. Second, we analyze the stability of the equilibrium points of the system in Fig. 1 and characterize those equilibrium points that are asymptotically stable. We consequently show that the equilibrium points found under the existence condition derived above are asymptotically stable; that is, the first contribution provides an existence condition of asymptotically stable equilibrium points. This result and the above discussion enable us to conclude that, when the PV current is below a certain level, the small-signal stability is preserved even though the PV generator is introduced.

Before closing this section, we give three remarks on this paper.
First, we note the difference between the results of this paper and those of previous studies. Four previous studies have considered the impact of PV penetration on the smallsignal stability $[2,3,8,17]$. In these studies, the authors have used system models given by the linearization around certain operating points, i.e., equilibrium points. That is, they have implicitly assumed that the target systems have equilibrium points. Contrary to this, we focus on the loss of equilibrium points as the impact of $\mathrm{PV}$ penetration on power systems and derive a condition for the existence of equilibrium points. In addition, we clarify the relation between the existence condition and the stability of equilibrium points. In these respects, the contributions of this paper are distinct from those of the previous studies.

Second, this paper presents an analytical result on the small-signal stability by considering the simplified system in Fig. 1. In the field of power engineering, researchers have often focused on stability analysis methods based on numerical computation and have applied their proposed methods to specific systems (see, e.g., $[3,8,17])$. Thus, for other systems with different parameter values, we cannot obtain any insight unless the analysis methods are again used. In addition, to investigate the effects of the parameter values on the systems, we have to use the analysis methods for various parameter values, which requires considerable time and effort. By contrast, this paper presents a stability condition as inequalities with respect to the parameters of the system in Fig. 1. As a result, we clarify that the system in Fig. 1 has the property mentioned above. This property is essential in the sense that it holds regardless of the values of the system parameters. Moreover, our stability condition allows us to estimate the magnitude of the PV current such that the small-signal stability is guaranteed, in advance. This is useful to construct a $\mathrm{PV}$-integrated power system that is small-signal stable. The analytical result of this paper has these advantages, compared with results based on numerical computation.

Finally, this paper is based on our conference paper [4], but differs from it in the following points. First, this paper includes the full explanations and the rigorous proofs of the main results, omitted from [4]. Second, we ensure that the paper is self-contained by showing the detailed derivation of the dynamics of the target system given in the Japanese paper [10]. Third, we present additional numerical examples to verify our result.

Notation Let $\mathbb{R}, \mathbb{R}_{+}$, and $\mathbb{R}_{0+}$ be the real number field, the set of positive real numbers, and the set of nonnegative real numbers, respectively. We use 0 to represent both the zero scalar and the zero vector. We denote by $a \angle \theta$ the complex number with the absolute value $a$ and the argument $\theta$. Finally, the following properties [18] for trigonometric functions and their inverses are used in this paper: 


$$
\begin{aligned}
& \sin \left(\cos ^{-1} x\right)=\sqrt{1-x^{2}} \\
& \sin \left(\tan ^{-1} y\right)=\frac{y}{\sqrt{1+y^{2}}} \\
& \cos \left(\cos ^{-1} x\right)=x \\
& \cos \left(\tan ^{-1} y\right)=\frac{1}{\sqrt{1+y^{2}}}
\end{aligned}
$$

where $-1 \leq x \leq 1$ and $-\infty \leq y \leq \infty$.

\section{Problem formulation}

\subsection{System description}

Consider the power system $\Sigma$ in Fig. 1, composed of the synchronous generator $G_{S}$, the PV generator $G_{P}$, and an infinite bus.

For the power system $\Sigma$, we impose the following assumptions.

- The phase angle of the current of $G_{P}$ for the infinite bus voltage tracks $\delta_{B}$, i.e., the phase angle of the voltage of the bus to which $G_{P}$ is connected.

- The magnitude of the PV current is constant.

The former implies that $G_{P}$ has a power conditioning unit. The latter is satisfied if the dynamics of $G_{S}$ is sufficiently faster than that of $G_{P}$. In addition, we regard $G_{S}$ as the combination of a voltage source and reactance.

Then, the dynamics of the system $\Sigma$ (i.e., the generator $\left.G_{S}\right)$ is described by

$M \ddot{\delta}_{S}(t)=P_{m}-P_{e}\left(\delta_{S}(t)\right)-D \dot{\delta}_{S}(t)$

where $\delta_{S}(t) \in \mathbb{R}$ is the phase angle of the voltage of $G_{S}$ for the infinite bus voltage, $M \in \mathbb{R}_{+}$is the moment of inertia, $P_{m} \in \mathbb{R}$ is the mechanical input, and $D \in \mathbb{R}_{+}$is the damping coefficient. The variable $P_{e}\left(\delta_{S}(t)\right) \in \mathbb{R}$ is the electrical output defined as

$$
\begin{aligned}
& P_{e}\left(\delta_{S}(t)\right) \\
& :=\frac{v_{S} v_{\infty} \sin \delta_{S}(t)-r_{L} v_{S} i_{P} \cos \left(\delta_{S}(t)-\delta_{B}\left(\delta_{S}(t)\right)\right)}{r_{S}+r_{L}},
\end{aligned}
$$

where $v_{S}, v_{\infty} \in \mathbb{R}_{+}$are the magnitude of the voltages of $G_{S}$ and the infinite bus, $r_{S}, r_{L} \in \mathbb{R}_{+}$are the reactance of $G_{S}$ and the transmission line, $i_{P} \in \mathbb{R}_{0+}$ is the magnitude of the current of $G_{P}$, and

$$
\begin{aligned}
& \delta_{B}\left(\delta_{S}(t)\right):= \\
& \begin{cases}\cos ^{-1}\left(\frac{-r_{S} r_{L} i_{P}}{\sqrt{\left(r_{L} v_{S} \sin \delta_{S}(t)\right)^{2}+\left(r_{L} v_{S} \cos \delta_{S}(t)+r_{S} v_{\infty}\right)^{2}}}\right) & \text { if } \sin \delta_{S}(t) \neq 0, \\
-\phi\left(\delta_{S}(t)\right) & \text { if } \sin \delta_{S}(t)=0, \\
\sin ^{-1}\left(\frac{r_{S} r_{L} i_{P}}{r_{L} v_{S} \cos \delta_{S}(t)+r_{S} v_{\infty}}\right) & \end{cases}
\end{aligned}
$$

for

$$
\begin{aligned}
& \phi\left(\delta_{S}(t)\right):= \\
& \begin{cases}\tan ^{-1}\left(\frac{r_{L} v_{S} \cos \delta_{S}(t)+r_{S} v_{\infty}}{r_{L} v_{S} \sin \delta_{S}(t)}\right) & \text { if } \sin \delta_{S}(t)>0, \\
\pi+\tan ^{-1}\left(\frac{r_{L} v_{S} \cos \delta_{S}(t)+r_{S} v_{\infty}}{r_{L} v_{S} \sin \delta_{S}(t)}\right) & \text { if } \sin \delta_{S}(t)<0 .\end{cases}
\end{aligned}
$$

Equation (5) is given as the swing equation. The derivations of (6) and (7) are based on [10]. The details are given in Appendix A.

\subsection{Stability analysis problem}

\subsubsection{Motivating examples}

Based on [6], we select the system parameters as $M:=$ $0.0186 \mathrm{~s}^{2}, P_{m}:=1.15 \mathrm{pu}, D:=0.00531 \mathrm{~s}, v_{S}:=1.12 \mathrm{pu}$, $v_{\infty}:=0.995 \mathrm{pu}, r_{S}:=0.300 \mathrm{pu}$, and $r_{L}:=0.500 \mathrm{pu}$. Figure 2 shows the time responses of the system $\Sigma$ with $i_{P}:=$ $0.2 \mathrm{pu}$ and $i_{P}:=0.5 \mathrm{pu}$, where $\left(\delta_{S}(0), \dot{\delta}_{S}(0)\right):=(1,0)$. We see that $\delta_{S}(t)$ with $i_{P}:=0.2$ pu converges but that with $i_{P}:=0.5 \mathrm{pu}$ does not.

To clarify the reason, we focus on the equilibrium points of the system $\Sigma$. Let $\left[\delta_{S}(t) \dot{\delta}_{S}(t)\right]^{\top}$ be the state variable vector. Then, (5) yields the following state equation of $\Sigma$ :

$\frac{d}{d t}\left[\begin{array}{l}\delta_{S}(t) \\ \dot{\delta}_{S}(t)\end{array}\right]=\left[\begin{array}{c}\dot{\delta}_{S}(t) \\ \frac{1}{M}\left(P_{m}-P_{e}\left(\delta_{S}(t)\right)-D \dot{\delta}_{S}(t)\right)\end{array}\right]$

From (9), the equilibrium points of $\Sigma$ are of the form $\left[\delta_{S}^{\star} 0\right]^{\top}$ where $\delta_{S}^{\star} \in \mathbb{R}$ is a solution to

$P_{m}=P_{e}\left(\delta_{S}\right)$

Thus, if the curve of $P_{e}\left(\delta_{S}\right)$ in (6) intersects with the straight line $P_{e}\left(\delta_{S}\right)=P_{m}$, then there exists an equilibrium point of $\Sigma$. In Fig. 3, the plots of $P_{e}\left(\delta_{S}\right)$ with $i_{P}:=0.2 \mathrm{pu}$ and $i_{P}:=0.5 \mathrm{pu}$ and the straight line $P_{e}\left(\delta_{S}\right)=P_{m}$ are depicted by the solid and dotted curves and the thin line, respectively. We see that the curve of $P_{e}\left(\delta_{S}\right)$ with $i_{P}:=0.2$ pu intersects 


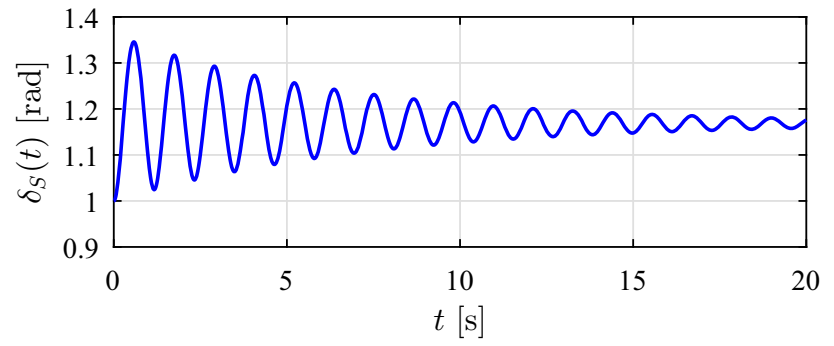

(a) $i_{P}:=0.2 \mathrm{pu}$

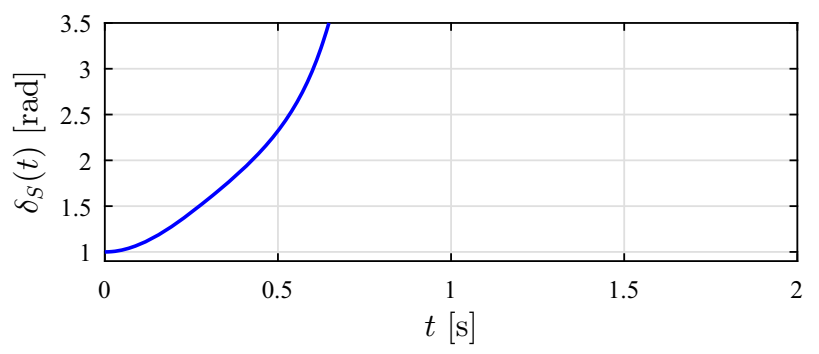

(b) $i_{P}:=0.5 \mathrm{pu}$

Fig. 2 Time responses of $\Sigma$ with $i_{P}:=0.2 \mathrm{pu}$ and $i_{P}:=0.5 \mathrm{pu}$

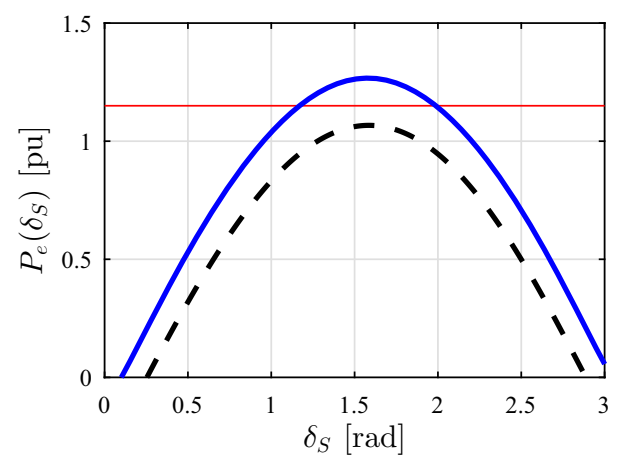

Fig. 3 Plots of $P_{e}\left(\delta_{S}\right)$ with $i_{P}:=0.2 \mathrm{pu}$ and $i_{P}:=0.5 \mathrm{pu}$

with the straight line $P_{e}\left(\delta_{S}\right)=P_{m}$ but that with $i_{P}:=0.5 \mathrm{pu}$ does not. This means that the existence of the equilibrium points of $\Sigma$ depends on $i_{P}$, which gives rise to the results in Fig. 2.

\subsubsection{Problem to be considered}

Motivated by these examples, we consider the following problem.

Problem 1 For the power system $\Sigma$,

(a) find a condition on $i_{P}$ for the existence of the equilibrium points;

(b) if an equilibrium point exists, then check its stability.

Remark 1 A straightforward method for solving Problem 1 is to directly solve (10) and clarify the properties of the equilib- rium points; however, solving (10) is difficult. This is because $P_{e}\left(\delta_{S}\right)$ contains the nonlinear term due to the presence of the PV generator $G_{P}$, i.e., $r_{L} v_{S} i_{P} \cos \left(\delta_{S}(t)-\delta_{B}\left(\delta_{S}(t)\right)\right)$ that includes trigonometric functions and their inverses, from (6) to (8). Although one may think that the system $\Sigma$ is simple, this fact makes the problem challenging.

\section{Existence of equilibrium points}

In this section, we address (a) in Problem 1. To this end, we first derive a condition to avoid the singular points of the system $\Sigma$. Based on this, we present a condition for the existence of the equilibrium points.

\subsection{Condition for avoiding singular points}

Because of $\cos ^{-1}(\cdot)$ and $\sin ^{-1}(\cdot)$ in (7), the system $\Sigma$ has singular points where the absolute values of the arguments of $\cos ^{-1}(\cdot)$ and $\sin ^{-1}(\cdot)$ are greater than one. We address this issue by presenting a condition on $i_{P}$ to avoid the singular points of $\Sigma$.

Lemma 1 If $i_{P}$ satisfies

$i_{P} \leq \frac{\left|r_{L} v_{S}-r_{S} v_{\infty}\right|}{r_{S} r_{L}}$

then the power system $\Sigma$ has no singular points, i.e.,

$\left|\frac{-r_{S} r_{L} i_{P}}{\sqrt{\left(r_{L} v_{S} \sin \delta_{S}\right)^{2}+\left(r_{L} v_{S} \cos \delta_{S}+r_{S} v_{\infty}\right)^{2}}}\right| \leq 1$,

for every $\delta_{S} \in \mathbb{R}$ satisfying $\sin \delta_{S} \neq 0$ and

$\left|\frac{r_{S} r_{L} i_{P}}{r_{L} v_{S} \cos \delta_{S}+r_{S} v_{\infty}}\right| \leq 1$

for every $\delta_{S} \in \mathbb{R}$ satisfying $\sin \delta_{S}=0$.

Proof The inequality (12) holds if

$\left(\frac{-r_{S} r_{L} i_{P}}{\sqrt{\left(r_{L} v_{S} \sin \delta_{S}\right)^{2}+\left(r_{L} v_{S} \cos \delta_{S}+r_{S} v_{\infty}\right)^{2}}}\right)^{2} \leq 1$

We can rewrite (14) as

$\left(r_{S} r_{L} i_{P}\right)^{2} \leq\left(r_{L} v_{S} \sin \delta_{S}\right)^{2}+\left(r_{L} v_{S} \cos \delta_{S}+r_{S} v_{\infty}\right)^{2}$,

which yields

$$
\begin{aligned}
i_{P} & \leq \frac{\sqrt{\left(r_{L} v_{S} \sin \delta_{S}\right)^{2}+\left(r_{L} v_{S} \cos \delta_{S}+r_{S} v_{\infty}\right)^{2}}}{r_{S} r_{L}} \\
& =\frac{\sqrt{r_{L}^{2} v_{S}^{2}+r_{S}^{2} v_{\infty}^{2}+2 r_{S} r_{L} v_{S} v_{\infty} \cos \delta_{S}}}{r_{S} r_{L}}
\end{aligned}
$$


because of $r_{S}, r_{L} \in \mathbb{R}_{+}$and $i_{P} \in \mathbb{R}_{0+}$. Since $\cos \delta_{S} \geq-1$ for every $\delta_{S} \in \mathbb{R}$, the right-hand side of (15) is greater than or equal to $\left(\sqrt{r_{L}^{2} v_{S}^{2}+r_{S}^{2} v_{\infty}^{2}-2 r_{S} r_{L} v_{S} v_{\infty}}\right) /\left(r_{S} r_{L}\right)$ for every $\delta_{S} \in \mathbb{R}$. Thus, we obtain

$$
\begin{aligned}
i_{P} & \leq \frac{\sqrt{r_{L}^{2} v_{S}^{2}+r_{S}^{2} v_{\infty}^{2}-2 r_{S} r_{L} v_{S} v_{\infty}}}{r_{S} r_{L}} \\
& =\frac{\sqrt{\left(r_{L} v_{S}-r_{S} v_{\infty}\right)^{2}}}{r_{S} r_{L}}
\end{aligned}
$$

which implies that (12) holds for every $\delta_{S} \in \mathbb{R}$ under (11). In addition, by choosing $\delta_{S}$ such that $\sin \delta_{S}=0$ in (12), we can show that (13) holds for every $\delta_{S} \in \mathbb{R}$ satisfying $\sin \delta_{S}=0$ under (11). This completes the proof.

\subsection{Main result}

Now, we derive the condition under which the equilibrium points of the system $\Sigma$ exist.

As explained in Sect. 2.2.1, $\Sigma$ has an equilibrium point if (10) has a solution. From (6)-(8) and Lemma $1, P_{e}\left(\delta_{S}\right)$ is a continuous function for $\delta_{S} \in(0, \pi)$ under (11). Therefore, it follows from the intermediate value theorem that if there exist a $\delta_{S}^{-} \in(0, \pi)$ and a $\delta_{S}^{+} \in\left(\delta_{S}^{-}, \pi\right)$ satisfying

$$
\begin{aligned}
& P_{e}\left(\delta_{S}^{-}\right)<P_{m}, \\
& P_{e}\left(\delta_{S}^{+}\right)>P_{m},
\end{aligned}
$$

then there exists at least one equilibrium point on $\left(\delta_{S}^{-}, \delta_{S}^{+}\right) \times$ $\{0\}$ (where $\{0\}$ corresponds to $\dot{\delta}_{S}=0$ ), as illustrated in Fig. 4 . Hence, we derive a condition on $i_{P}$ to achieve (16) and (17) for given $\delta_{S}^{-}$and $\delta_{S}^{+}$.

Based on this idea, we obtain the following result.

Theorem 1 For the power system $\Sigma$, assume that (11) holds. If there exist $a \delta_{S}^{-} \in(0, \pi / 2)$ and $a \delta_{S}^{+} \in\left(\delta_{S}^{-}, \pi / 2\right]$ satisfying the following two conditions, then there exists at least one equilibrium point on $\left(\delta_{S}^{-}, \delta_{S}^{+}\right) \times\{0\}$.

(C1) $c_{0}\left(\delta_{S}^{-}\right)<0$ and $c_{0}\left(\delta_{S}^{+}\right)>0$,

(C2) $i_{P}<\alpha_{21}\left(\delta_{S}^{+}\right)$,

where

$$
\begin{aligned}
c_{0}\left(\delta_{S}\right) & :=\frac{v_{S} v_{\infty} \sin \delta_{S}}{r_{S}+r_{L}}-P_{m}, \\
\alpha_{21}\left(\delta_{S}\right) & :=\frac{-c_{12}\left(\delta_{S}\right)-\sqrt{c_{12}^{2}\left(\delta_{S}\right)-4 c_{2}\left(\delta_{S}\right) c_{0}\left(\delta_{S}\right)}}{2 c_{2}\left(\delta_{S}\right)},
\end{aligned}
$$

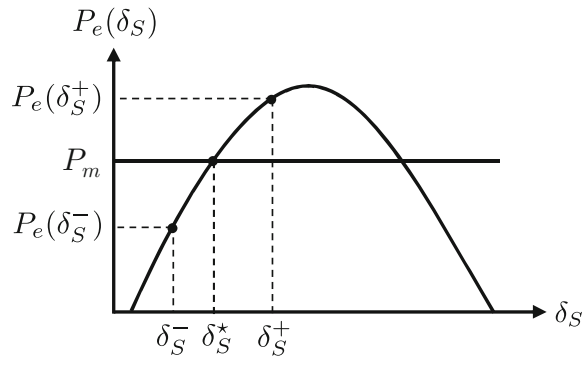

Fig. 4 Idea of analysis

for

$c_{12}\left(\delta_{S}\right):=\frac{-r_{L} v_{S}\left(r_{L} v_{S}+r_{S} v_{\infty} \cos \delta_{S}\right)}{\left(r_{S}+r_{L}\right) \sqrt{f\left(\delta_{S}\right)}}$,

$c_{2}\left(\delta_{S}\right):=\frac{-r_{S}^{2} r_{L}^{2} v_{S} v_{\infty} \sin \delta_{S}}{\left(r_{S}+r_{L}\right) f\left(\delta_{S}\right)}$,

$f\left(\delta_{S}\right):=r_{L}^{2} v_{S}^{2}+r_{S}^{2} v_{\infty}^{2}+2 r_{S} r_{L} v_{S} v_{\infty} \cos \delta_{S}$.

Proof Using a trigonometric addition formula for (6) gives

$$
\begin{aligned}
& P_{e}\left(\delta_{S}\right)=\frac{v_{S} v_{\infty} \sin \delta_{S}}{r_{S}+r_{L}} \\
& -\frac{r_{L} v_{S} i_{P}\left(\cos \delta_{S} \cos \left(\delta_{B}\left(\delta_{S}\right)\right)+\sin \delta_{S} \sin \left(\delta_{B}\left(\delta_{S}\right)\right)\right)}{r_{S}+r_{L}} .
\end{aligned}
$$

If $\delta_{S} \in(0, \pi / 2]$, then $\sin \delta_{S}>0$. Furthermore, from Lemma 1 , (12) holds for every $\delta_{S} \in(0, \pi / 2]$ under (11). Thus, by substituting the first equations of (7) and (8) into (23) and using (1)-(4), we obtain

$P_{e}\left(\delta_{S}\right)=c_{2}\left(\delta_{S}\right) i_{P}^{2}+c_{1}\left(\delta_{S}, i_{P}\right) i_{P}+c_{0}\left(\delta_{S}\right)+P_{m}$,

where

$c_{1}\left(\delta_{S}, i_{P}\right)$

$:=-\frac{r_{L} v_{S}\left(r_{L} v_{S}+r_{S} v_{\infty} \cos \delta_{S}\right) \sqrt{f\left(\delta_{S}\right)-r_{S}^{2} r_{L}^{2} i_{P}^{2}}}{\left(r_{S}+r_{L}\right) f\left(\delta_{S}\right)}$.

First, we derive a condition corresponding to (16). Based on (11), we substitute $i_{P}=\left|r_{L} v_{S}-r_{S} v_{\infty}\right| /\left(r_{S} r_{L}\right)$ into the right-hand side of (25), which provides

$$
\begin{aligned}
c_{11}\left(\delta_{S}\right):= & -\frac{r_{L} v_{S}\left(r_{L} v_{S}+r_{S} v_{\infty} \cos \delta_{S}\right)}{r_{S}+r_{L}} \\
& \times \frac{\sqrt{2 r_{S} r_{L} v_{S} v_{\infty}\left(\cos \delta_{S}+1\right)}}{f\left(\delta_{S}\right)} .
\end{aligned}
$$

By the definition, $f\left(\delta_{S}\right)$ is positive for every $\delta_{S} \in(0, \pi / 2]$ because of $\cos \delta_{S} \geq 0$. This and $\cos \delta_{S} \geq 0$ (for every $\delta_{S} \in$ $(0, \pi / 2])$ imply that $c_{1}\left(\delta_{S}, i_{P}\right) \leq c_{11}\left(\delta_{S}\right)$ for every $i_{P} \in \mathbb{R}_{0+}$ 
satisfying (11) if $\delta_{S} \in(0, \pi / 2]$. Thus, it follows from (24) that

$P_{e}\left(\delta_{S}\right) \leq c_{2}\left(\delta_{S}\right) i_{P}^{2}+c_{11}\left(\delta_{S}\right) i_{P}+c_{0}\left(\delta_{S}\right)+P_{m}$,

for every $i_{P} \in \mathbb{R}_{0+}$ satisfying (11) subject to $\delta_{S} \in(0, \pi / 2]$. Replacing $\delta_{S}$ in (27) with $\delta_{S}^{-}$gives

$c_{2}\left(\delta_{S}^{-}\right) i_{P}^{2}+c_{11}\left(\delta_{S}^{-}\right) i_{P}+c_{0}\left(\delta_{S}^{-}\right)<0$,

as a sufficient condition for (16).

Second, we derive a condition corresponding to (17). Based on $i_{P} \in \mathbb{R}_{0+}$, we substitute $i_{P}=0$ into the right-hand side of (25), which provides $c_{12}\left(\delta_{S}\right)$ in (20). Then, similar to the first case, we can show that $c_{1}\left(\delta_{S}, i_{P}\right) \geq c_{12}\left(\delta_{S}\right)$ for every $i_{P} \in \mathbb{R}_{0+}$ satisfying (11) under $\delta_{S} \in(0, \pi / 2]$. Applying this to (24) yields

$P_{e}\left(\delta_{S}\right) \geq c_{2}\left(\delta_{S}\right) i_{P}^{2}+c_{12}\left(\delta_{S}\right) i_{P}+c_{0}\left(\delta_{S}\right)+P_{m}$,

for every $i_{P} \in \mathbb{R}_{0+}$ satisfying (11) subject to $\delta_{S} \in(0, \pi / 2]$. Replacing $\delta_{S}$ in (29) with $\delta_{S}^{+}$gives

$c_{2}\left(\delta_{S}^{+}\right) i_{P}^{2}+c_{12}\left(\delta_{S}^{+}\right) i_{P}+c_{0}\left(\delta_{S}^{+}\right)>0$,

as a sufficient condition for (17).

In summary, if (28) and (30) hold, then (16) and (17) hold. Thus, if (28) and (30) hold for a $\delta_{S}^{-} \in(0, \pi / 2)$ and a $\delta_{S}^{+} \in\left(\delta_{S}^{-}, \pi / 2\right]$, then there exists at least one equilibrium point on $\left(\delta_{S}^{-}, \delta_{S}^{+}\right) \times\{0\}$ as described at the beginning of this section. Inequalities (28) and (30) have a solution under (C1), which is expressed as $(\mathrm{C} 2)$. The derivation of $(\mathrm{C} 2)$ is shown in Appendix B. This completes the proof.

Theorem 1 gives a sufficient condition on $i_{P}$ such that the system $\Sigma$ has equilibrium points. Condition (C1) guarantees that there exists at least one equilibrium point on $\left(\delta_{S}^{-}, \delta_{S}^{+}\right) \times$ $\{0\}$ in the case of $i_{P}:=0$. In fact, by substituting $i_{P}=0$ to (6) and considering (18), we can show that $c_{0}\left(\delta_{S}^{-}\right)<0$ and $c_{0}\left(\delta_{S}^{+}\right)>0$ correspond to (16) and (17), respectively. On the other hand, condition (C2) holds when $i_{P}$ is sufficiently small. In fact, since $\alpha_{21}\left(\delta_{S}^{+}\right)$is a positive real number as shown in Lemma 3 in Appendix $\mathrm{B}$, there exists an $i_{P}>0$ satisfying $(\mathrm{C} 2)$ for a given $\delta_{S}^{+}$. Hence, Theorem 1 means that if the power system given by removing $G_{P}$ from $\Sigma$ has equilibrium points, then the existence of the equilibrium points of $\Sigma$ is guaranteed as long as $i_{P}$ is sufficiently small.

We comment on the choice of $\delta_{S}^{-}$and $\delta_{S}^{+}$. To use Theorem 1 , we need to appropriately choose a $\delta_{S}^{-} \in(0, \pi / 2)$ and a $\delta_{S}^{+} \in\left(\delta_{S}^{-}, \pi / 2\right]$. A good choice is to let $\delta_{S}^{-}$and $\delta_{S}^{+}$be a sufficiently small positive number and $\pi / 2$, respectively. The reason is that finding the equilibrium points becomes easier as the difference between $\delta_{S}^{+}$and $\delta_{S}^{-}$increases as shown in
Fig. 4. In this method, we only have to check (C1) and (C2) for one pair consisting of a $\delta_{S}^{-}$and a $\delta_{S}^{+}$.

\subsection{Example}

Consider again the system $\Sigma$ handled in Sect. 2.2.1, where $i_{P}:=0.2 \mathrm{pu}$. Then, (11) in Lemma 1 is calculated as $i_{P} \leq$ $1.74 \mathrm{pu}$ and thus is satisfied. For $\delta_{S}^{-}:=0.01$ and $\delta_{S}^{+}:=\pi / 2$, (18) provides $c_{0}\left(\delta_{S}^{-}\right)=-1.14$ and $c_{0}\left(\delta_{S}^{+}\right)=0.243$, from which condition (C1) in Theorem 1 holds. Moreover, since $\alpha_{21}\left(\delta_{S}^{+}\right)$is calculated as $\alpha_{21}\left(\delta_{S}^{+}\right)=0.375$ from (19), condition $(\mathrm{C} 2)$ is $i_{P}<0.375 \mathrm{pu}$ and $i_{P}:=0.2 \mathrm{pu}$ satisfies it. Hence, there exists at least one equilibrium point on $(0.01, \pi / 2) \times\{0\}$ from Theorem 1 .

Meanwhile, we see from Fig. 3 that when $i_{P}:=0.2 \mathrm{pu}$, the system $\Sigma$ has an equilibrium point on $(0.01, \pi / 2) \times\{0\}$, i.e., $\left[\begin{array}{ll}1.17 & 0\end{array}\right]^{\top}$, which demonstrates the above result.

\section{Stability of equilibrium points}

Next, we address (b) in Problem 1. That is, we analyze the stability of the equilibrium points of the system $\Sigma$.

\subsection{Main result}

We introduce the new state variable vector $x(t):=\left[\delta_{S}(t)-\right.$ $\left.\delta_{S}^{\star} \quad \dot{\delta}_{S}(t)\right]^{\top} \in \mathbb{R}^{2}$ to shift the equilibrium point $\left[\begin{array}{ll}\delta_{S}^{\star} & 0\end{array}\right]^{\top}$ to $x=0$. Although the system $\Sigma$ is a hybrid system from (7) and (8), if $\sin \delta_{S}^{\star}>0$, then there exists a set containing $x=0$ on which $\Sigma$ can be regarded as a non-hybrid system. In this case, it follows from (6), the first equations of (7) and (8), and (9) that the linearized system around $x=0$ is expressed as

$\dot{x}(t)=A x(t)$,

where

$A:=\left[\begin{array}{cc}0 & 1 \\ a_{21}\left(\delta_{S}^{\star}\right) & -\frac{D}{M}\end{array}\right]$,

for

$$
\begin{aligned}
a_{21}\left(\delta_{S}^{\star}\right):= & -\frac{v_{S} v_{\infty} \cos \delta_{S}^{\star}}{M\left(r_{S}+r_{L}\right)}-\frac{r_{L} v_{S} i_{P} \sin \left(\delta_{S}^{\star}-\delta_{B}\left(\delta_{S}^{\star}\right)\right)}{M\left(r_{S}+r_{L}\right)} \\
& \times\left(1-\frac{1}{f\left(\delta_{S}^{\star}\right)}\left(\frac{r_{S}^{2} r_{L}^{2} v_{S} v_{\infty} i_{P} \sin \delta_{S}^{\star}}{\sqrt{f\left(\delta_{S}^{\star}\right)-r_{S}^{2} r_{L}^{2} i_{P}^{2}}}\right.\right. \\
& \left.\left.+r_{L}^{2} v_{S}^{2}+r_{S} r_{L} v_{S} v_{\infty} \cos \delta_{S}^{\star}\right)\right) .
\end{aligned}
$$


An investigation of the eigenvalues of the matrix $A$ enables us to analyze the stability of the equilibrium point $\left[\begin{array}{ll}\delta_{S}^{\star} & 0\end{array}\right]^{\top}$ (satisfying $\sin \delta_{S}^{\star}>0$ ).

As a result, we obtain the following theorem.

Theorem 2 For the power system $\Sigma$, assume that (11) holds and there exists the equilibrium point $\left[\begin{array}{ll}\delta_{S}^{\star} & 0\end{array}\right]^{\top}$ satisfying $\sin \delta_{S}^{\star}>0$. Then, the equilibrium point is asymptotically stable if $\delta_{S}^{\star} \in(0, \pi / 2)$.

Proof From (32), the eigenvalues $\lambda_{1}$ and $\lambda_{2}$ of $A$ are given by

$$
\begin{aligned}
& \lambda_{1}=\frac{-D-\sqrt{D^{2}+4 a_{21}\left(\delta_{S}^{\star}\right) M}}{2 M}, \\
& \lambda_{2}=\frac{-D+\sqrt{D^{2}+4 a_{21}\left(\delta_{S}^{\star}\right) M}}{2 M} .
\end{aligned}
$$

Therefore, it follows from $M, D \in \mathbb{R}_{+}$that the equilibrium point $\left[\begin{array}{ll}\delta_{S}^{\star} & 0\end{array}\right]^{\top}$ is asymptotically stable if $a_{21}\left(\delta_{S}^{\star}\right)<0$. The proof of $a_{21}\left(\delta_{S}^{\star}\right)<0$ is shown in Appendix C, which proves the theorem.

Theorem 2 characterizes the asymptotically stable equilibrium points of the system $\Sigma$. This result means that the equilibrium points found by Theorem 1 are asymptotically stable. That is, Theorem 1 presents a sufficient condition for the existence of the asymptotically stable equilibrium points.

\subsection{Examples}

Consider again the system $\Sigma$ handled in Sect. 2.2.1. As given in Sect. 3.3, a condition for the existence of the equilibrium points is $i_{P}<0.375 \mathrm{pu}$.

For $i_{P}:=0.1,0.2,0.3 \mathrm{pu}$ satisfying the above condition, we calculate $\delta_{S}^{\star}$ in the interval $(0, \pi)$ by numerically solving (10) and then obtain $\lambda_{1}$ and $\lambda_{2}$ in (34) and (35). The result is summarized in Table 1 where $j:=\sqrt{-1}$. We see that the equilibrium points satisfying $\delta_{S}^{\star} \in(0, \pi / 2)$ are asymptotically stable because the real parts of the corresponding $\lambda_{1}$ and $\lambda_{2}$ are negative. This demonstrates Theorem 2 .

As an example of the time response of the system $\Sigma$, that with $i_{P}:=0.3 \mathrm{pu}$ is presented in Fig. 5, where $\left(\delta_{S}(0), \dot{\delta}_{S}(0)\right):=(1.2,-0.3)$ and the thin line expresses $\delta_{S}=1.31$, i.e., $\delta_{S}^{\star}$ in the asymptotically stable equilibrium point. It turns out that $\delta_{S}(t)$ converges to $\delta_{S}^{\star}$.

\section{Conclusion}

This paper has examined the existence of equilibrium points and their stability for a power system with a PV generator. By focusing on a property of the equilibrium points and using
Table 1 Stability of equilibrium points (o: asymptotically stable; $x$ : unstable)

\begin{tabular}{lllll}
\hline$i_{P}[\mathrm{pu}]$ & $\delta_{S}^{\star}$ & $\lambda_{1}$ & $\lambda_{2}$ & Stability \\
\hline 0.1 & 1.06 & $-0.143-6.07 j$ & $-0.143+6.07 j$ & $\circ$ \\
& 2.09 & -6.23 & 5.95 & $\times$ \\
0.2 & 1.17 & $-0.143-5.48 j$ & $-0.143+5.48 j$ & $\circ$ \\
& 1.99 & -5.64 & 5.36 & $\times$ \\
0.3 & 1.31 & $-0.143-4.49 j$ & $-0.143+4.49 j$ & $\circ$ \\
& 1.85 & -4.65 & 4.37 & $\times$ \\
\hline
\end{tabular}

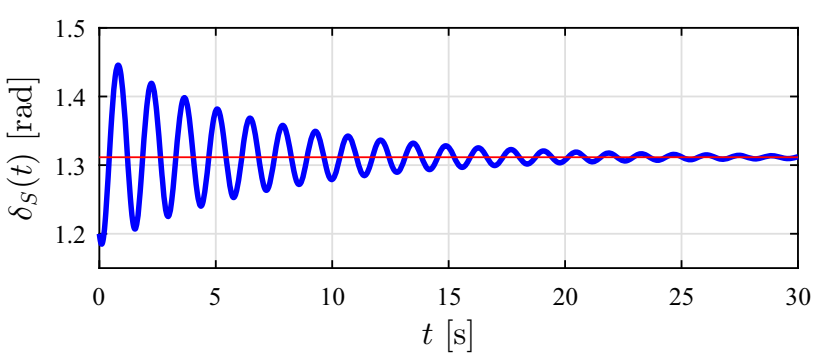

Fig. 5 Time response of $\Sigma$ with $i_{P}:=0.3 \mathrm{pu}$

the intermediate value theorem, we have derived a condition under which the equilibrium points exist. Furthermore, we have characterized the asymptotically stable equilibrium points and have proven that these points are found by the existence condition. These results not only clarify the effect of PV penetration on small-signal stability, but are also useful to construct a power system with a PV generator with asymptotically stable equilibrium points.

Although this paper has presented a solution to the stability analysis problem, other problems remain to be addressed. For example, it would be necessary to extend our result to power systems consisting of multiple synchronous generators and PV generators. In addition, our result would have to be extended to controller design for power systems with PV generators.

Open Access This article is distributed under the terms of the Creative Commons Attribution 4.0 International License (http://creativecomm ons.org/licenses/by/4.0/), which permits unrestricted use, distribution, and reproduction in any medium, provided you give appropriate credit to the original author(s) and the source, provide a link to the Creative Commons license, and indicate if changes were made.

\section{A Derivations of (6) and (7) [10]}

Let $a e^{j \theta}$ be the complex number with the absolute value $a$ and the argument $\theta$, where $e^{j \theta}=\cos \theta+j \sin \theta$. In addition, let $\bar{z}$ be the complex conjugate of the complex number $z$. 
Consider the power system $\Sigma$ in Fig. 1. We have

$\frac{v_{S} e^{j \delta_{S}}-v_{B} e^{j \delta_{B}}}{j r_{S}}+i_{P} e^{j \delta_{B}}=\frac{v_{B} e^{j \delta_{B}}-v_{\infty}}{j r_{L}}$,

as a circuit equation. This can be rewritten as

$v_{B}=\frac{r_{L} v_{S} e^{j\left(\delta_{S}-\delta_{B}\right)}+j r_{S} r_{L} i_{P}+r_{S} v_{\infty} e^{-j \delta_{B}}}{r_{S}+r_{L}}$.

Thus, the complex power of the generator $G_{S}$ is given by

$$
\begin{array}{r}
v_{S} e^{j \delta_{S}} \frac{v_{S} e^{-j \delta_{S}}-\overline{v_{B}} e^{-j \delta_{B}}}{-j r_{S}} \\
=\frac{v_{S} v_{\infty} \sin \delta_{S}-r_{L} v_{S} i_{P} \cos \left(\delta_{S}-\delta_{B}\left(\delta_{S}\right)\right)}{r_{S}+r_{L}} \\
+j\left(-\frac{v_{S} v_{\infty} \cos \delta_{S}+r_{L} v_{S} i_{P} \sin \left(\delta_{S}-\delta_{B}\left(\delta_{S}\right)\right)}{r_{S}+r_{L}}\right. \\
\left.+\frac{v_{S}^{2}}{r_{S}}-\frac{r_{L} v_{S}^{2}}{r_{S}\left(r_{L}+r_{S}\right)}\right) .
\end{array}
$$

By focusing on the real part of the right-hand side of (38), we obtain (6).

Next, we derive (7). From $v_{B} \in \mathbb{R}_{+}, \delta_{B}$ is determined so that the imaginary part of the right-hand side of (37) is equal to zero. Hence, we obtain

$$
\begin{aligned}
r_{L} & v_{S} \sin \left(\delta_{S}-\delta_{B}\right)+r_{S} r_{L} i_{P}+r_{S} v_{\infty} \sin \left(-\delta_{B}\right) \\
= & r_{L} v_{S}\left(\sin \delta_{S} \cos \delta_{B}-\cos \delta_{S} \sin \delta_{B}\right) \\
& +r_{S} r_{L} i_{P}-r_{S} v_{\infty} \sin \delta_{B} \\
= & r_{L} v_{S} \sin \delta_{S} \cos \delta_{B} \\
& -\left(r_{L} v_{S} \cos \delta_{S}+r_{S} v_{\infty}\right) \sin \delta_{B}+r_{S} r_{L} i_{P} \\
= & 0
\end{aligned}
$$

where the first equality is given by a trigonometric addition formula. For (39), we consider the following two cases.

(a) Case of $\sin \delta_{S} \neq 0$ : Combining $\cos \delta_{B}$ and $\sin \delta_{B}$ in (39) yields

$$
\begin{aligned}
& \sqrt{\left(r_{L} v_{S} \sin \delta_{S}\right)^{2}+\left(r_{L} v_{S} \cos \delta_{S}+r_{S} v_{\infty}\right)^{2}} \\
& \times \cos \left(\delta_{B}+\phi\left(\delta_{S}\right)\right)=-r_{S} r_{L} i_{P} .
\end{aligned}
$$

Equation (40) implies the first equation of (7).

(b) Case of $\sin \delta_{S}=0$ : By substituting $\sin \delta_{S}=0$ into (39), we obtain the second equation of (7).

From (a) and (b), (7) is derived.

\section{B Derivation of (C2) in Theorem 1}

\section{B.1 Preliminary}

We prepare the following lemma.

Lemma 2 Consider $c_{12}\left(\delta_{S}\right), c_{2}\left(\delta_{S}\right)$, and $c_{11}\left(\delta_{S}\right)$ in (20), (21), and (26). These are negative for every $\delta_{S} \in(0, \pi / 2]$.

Proof From (20)-(22), (26), and $\sin \delta_{S}>0$ and $\cos \delta_{S} \geq 0$ for every $\delta_{S} \in(0, \pi / 2]$, we prove the lemma.

Lemma 2 leads to the following result.

Lemma 3 Consider $\alpha_{21}\left(\delta_{S}\right)$ in (19). Let

$\begin{aligned} \alpha_{22}\left(\delta_{S}\right) & :=\frac{-c_{12}\left(\delta_{S}\right)+\sqrt{c_{12}^{2}\left(\delta_{S}\right)-4 c_{2}\left(\delta_{S}\right) c_{0}\left(\delta_{S}\right)}}{2 c_{2}\left(\delta_{S}\right)}, \\ \alpha_{11}\left(\delta_{S}\right):= & \frac{-c_{11}\left(\delta_{S}\right)-\sqrt{c_{11}^{2}\left(\delta_{S}\right)-4 c_{2}\left(\delta_{S}\right) c_{0}\left(\delta_{S}\right)}}{2 c_{2}\left(\delta_{S}\right)} .\end{aligned}$

Then, the following statements hold.

(a) For every $\delta_{S}^{+} \in(0, \pi / 2]$ satisfying $c_{0}\left(\delta_{S}^{+}\right)>0$ in $(C l)$ in Theorem 1, $\alpha_{21}\left(\delta_{S}^{+}\right)$and $\alpha_{22}\left(\delta_{S}^{+}\right)$are positive and negative real numbers, respectively.

(b) For every $\delta_{S}^{-} \in(0, \pi / 2)$ satisfying $c_{0}\left(\delta_{S}^{-}\right)<0$ in $(C 1)$ and $c_{11}^{2}\left(\delta_{S}^{-}\right)-4 c_{2}\left(\delta_{S}^{-}\right) c_{0}\left(\delta_{S}^{-}\right)>0, \alpha_{11}\left(\delta_{S}^{-}\right)$is a negative real number.

Proof (a) From $\delta_{S}^{+} \in(0, \pi / 2]$ and Lemma 2, we obtain $c_{12}\left(\delta_{S}^{+}\right)<0$ and $c_{2}\left(\delta_{S}^{+}\right)<0$. This and $c_{0}\left(\delta_{S}^{+}\right)>0$ imply

$-c_{12}\left(\delta_{S}^{+}\right)-\sqrt{c_{12}^{2}\left(\delta_{S}^{+}\right)-4 c_{2}\left(\delta_{S}^{+}\right) c_{0}\left(\delta_{S}^{+}\right)}<0$,

where $c_{12}^{2}\left(\delta_{S}^{+}\right)-4 c_{2}\left(\delta_{S}^{+}\right) c_{0}\left(\delta_{S}^{+}\right)>0$ is noted. Thus, it follows from (19) and $c_{2}\left(\delta_{S}^{+}\right)<0$ that $\alpha_{21}\left(\delta_{S}^{+}\right)$is a positive real number. Meanwhile, (41), $c_{12}\left(\delta_{S}^{+}\right)<0, c_{2}\left(\delta_{S}^{+}\right)<0$, and $c_{12}^{2}\left(\delta_{S}^{+}\right)-4 c_{2}\left(\delta_{S}^{+}\right) c_{0}\left(\delta_{S}^{+}\right)>0$ show that $\alpha_{22}\left(\delta_{S}^{+}\right)$is a negative real number. This completes the proof of (a).

(b) From $\delta_{S}^{-} \in(0, \pi / 2)$ and Lemma 2, we have $c_{2}\left(\delta_{S}^{-}\right)<$ 0 and $c_{11}\left(\delta_{S}^{-}\right)<0$. Therefore, it follows from $c_{0}\left(\delta_{S}^{-}\right)<0$ that

$-c_{11}\left(\delta_{S}^{-}\right)-\sqrt{c_{11}^{2}\left(\delta_{S}^{-}\right)-4 c_{2}\left(\delta_{S}^{-}\right) c_{0}\left(\delta_{S}^{-}\right)}>0$,

where $c_{11}^{2}\left(\delta_{S}^{-}\right)-4 c_{2}\left(\delta_{S}^{-}\right) c_{0}\left(\delta_{S}^{-}\right)>0$ is used. This, together with (42) and $c_{2}\left(\delta_{S}^{-}\right)<0$, proves (b).

Lemma 3 provides the properties of the solutions to the quadratic equations

$c_{2}\left(\delta_{S}^{-}\right) i_{P}^{2}+c_{11}\left(\delta_{S}^{-}\right) i_{P}+c_{0}\left(\delta_{S}^{-}\right)=0$, 
$c_{2}\left(\delta_{S}^{+}\right) i_{P}^{2}+c_{12}\left(\delta_{S}^{+}\right) i_{P}+c_{0}\left(\delta_{S}^{+}\right)=0$.

By using these properties, we derive (C2) in Theorem 1 in the next section.

\section{B.2 Main part}

Condition (C2) is given as a solution to the simultaneous inequalities of (28) and (30). In what follows, we solve each inequality and then show $(\mathrm{C} 2)$.

\section{B.2.1 Solution to (28)}

First, we consider (43). This has three types of solutions depending on the value of $c_{11}^{2}\left(\delta_{S}^{-}\right)-4 c_{2}\left(\delta_{S}^{-}\right) c_{0}\left(\delta_{S}^{-}\right)$. Hence, (28) also has three types of solutions, which are described as follows.

(a) Case of $c_{11}^{2}\left(\delta_{S}^{-}\right)-4 c_{2}\left(\delta_{S}^{-}\right) c_{0}\left(\delta_{S}^{-}\right)>0$ : Equation (43) has the two real solutions $i_{P}=\alpha_{11}\left(\delta_{S}^{-}\right)$and $i_{P}=$ $\alpha_{12}\left(\delta_{S}^{-}\right)$where

$$
\alpha_{12}\left(\delta_{S}\right):=\frac{-c_{11}\left(\delta_{S}\right)+\sqrt{c_{11}^{2}\left(\delta_{S}\right)-4 c_{2}\left(\delta_{S}\right) c_{0}\left(\delta_{S}\right)}}{2 c_{2}\left(\delta_{S}\right)} .
$$

We should notice that $\alpha_{12}\left(\delta_{S}^{-}\right)<\alpha_{11}\left(\delta_{S}^{-}\right)$from $c_{2}\left(\delta_{S}^{+}\right)<0$ in the proof of Lemma 3. This fact and $c_{2}\left(\delta_{S}^{+}\right)<0$ show that a solution to (28) is expressed as

$$
i_{P}<\alpha_{12}\left(\delta_{S}^{-}\right) \text {or } i_{P}>\alpha_{11}\left(\delta_{S}^{-}\right) .
$$

(b) Case of $c_{11}^{2}\left(\delta_{S}^{-}\right)-4 c_{2}\left(\delta_{S}^{-}\right) c_{0}\left(\delta_{S}^{-}\right)=0$ : The solution to (43) is $i_{P}=-c_{11}\left(\delta_{S}^{-}\right) /\left(2 c_{2}\left(\delta_{S}^{-}\right)\right)$. Therefore, it follows from $c_{2}\left(\delta_{S}^{-}\right)<0$ in the proof of Lemma 3 that $i_{P} \neq$ $-c_{11}\left(\delta_{S}^{-}\right) /\left(2 c_{2}\left(\delta_{S}^{-}\right)\right)$solves (28). This means that (28) holds for every $i_{P} \in \mathbb{R}_{0+}$ since $-c_{11}\left(\delta_{S}^{-}\right) /\left(2 c_{2}\left(\delta_{S}^{-}\right)\right)<$ 0 from $c_{11}\left(\delta_{S}^{-}\right)<0$ in the proof of Lemma 3 and $c_{2}\left(\delta_{S}^{-}\right)<0$.

(c) Case of $c_{11}^{2}\left(\delta_{S}^{-}\right)-4 c_{2}\left(\delta_{S}^{-}\right) c_{0}\left(\delta_{S}^{-}\right)<0$ : There are no real solutions to (43), which implies that (28) holds for every $i_{P} \in \mathbb{R}_{0+}$ because of $c_{2}\left(\delta_{S}^{-}\right)<0$.

\section{B.2.2 Solution to $(30)$}

We next consider (44). Since $c_{12}^{2}\left(\delta_{S}^{+}\right)-4 c_{2}\left(\delta_{S}^{+}\right) c_{0}\left(\delta_{S}^{+}\right)>0$ as shown in the proof of Lemma 3, (44) has the two real solutions $i_{P}=\alpha_{21}\left(\delta_{S}^{+}\right)$and $i_{P}=\alpha_{22}\left(\delta_{S}^{+}\right)$. This and $c_{2}\left(\delta_{S}^{+}\right)<0$ imply that

$\alpha_{22}\left(\delta_{S}^{+}\right)<i_{P}<\alpha_{21}\left(\delta_{S}^{+}\right)$

solves (30) because $\alpha_{22}\left(\delta_{S}^{+}\right)<\alpha_{21}\left(\delta_{S}^{+}\right)$from Lemma 3.

\section{B.2.3 Solution to (28) and (30)}

The solution to be obtained satisfies both (28) and (30). Thus, from the above discussion, we consider the following three cases.

(a) Case of $c_{11}^{2}\left(\delta_{S}^{-}\right)-4 c_{2}\left(\delta_{S}^{-}\right) c_{0}\left(\delta_{S}^{-}\right)>0$ : From (46) and (47), a solution to (28) and (30) is given by $i_{P}>\alpha_{11}\left(\delta_{S}^{-}\right)$ and $\alpha_{22}\left(\delta_{S}^{+}\right)<i_{P}<\alpha_{21}\left(\delta_{S}^{+}\right)$. Therefore, by noting $\alpha_{11}\left(\delta_{S}^{-}\right)<0, \alpha_{21}\left(\delta_{S}^{+}\right)>0$, and $\alpha_{22}\left(\delta_{S}^{+}\right)<0$ in Lemma 3 , we obtain $(\mathrm{C} 2)$ in Theorem 1.

(b) Case of $c_{11}^{2}\left(\delta_{S}^{-}\right)-4 c_{2}\left(\delta_{S}^{-}\right) c_{0}\left(\delta_{S}^{-}\right)=0$ : As mentioned in (b) in Appendix B.2.1, (28) holds for every $i_{P} \in \mathbb{R}_{0+}$. Hence, in a similar way to that in (a), we obtain (C2) as a solution to (28) and (30).

(c) Case of $c_{11}^{2}\left(\delta_{S}^{-}\right)-4 c_{2}\left(\delta_{S}^{-}\right) c_{0}\left(\delta_{S}^{-}\right)<0$ : Similar to (b), we obtain (C2) as a solution to (28) and (30).

From (a)-(c), we derive (C2) in Theorem 1.

\section{Proof of $a_{21}\left(\delta_{S}^{\star}\right)<0$ in proof of Theorem 2}

We show $a_{21}\left(\delta_{S}^{\star}\right)<0$ by considering the following three cases for $\sin \left(\delta_{S}^{\star}-\delta_{B}\left(\delta_{S}^{\star}\right)\right)$ in $a_{21}\left(\delta_{S}^{\star}\right)$ in (33).

\section{C.1 Case of $\sin \left(\delta_{S}^{\star}-\delta_{B}\left(\delta_{S}^{\star}\right)\right)>0$}

If $\delta_{S}^{\star} \in(0, \pi / 2)$, then $\sin \left(\delta_{S}^{\star}\right)>0$. Furthermore, Lemma 1 means that (12) holds for every $\delta_{S}^{\star} \in(0, \pi / 2)$ subject to (11). Thus, the first equations of (7) and (8), and (1)-(4) provide

$$
\begin{aligned}
\sin \left(\delta_{S}^{\star}-\delta_{B}\left(\delta_{S}^{\star}\right)\right)= & -\frac{r_{S} r_{L} i_{P}\left(r_{L} v_{S}+r_{S} v_{\infty} \cos \delta_{S}^{\star}\right)}{f\left(\delta_{S}^{\star}\right)} \\
& +\frac{r_{S} v_{\infty} \sin \delta_{S}^{\star} \sqrt{f\left(\delta_{S}^{\star}\right)-r_{S}^{2} r_{L}^{2} i_{P}^{2}}}{f\left(\delta_{S}^{\star}\right)} .
\end{aligned}
$$

From a similar discussion to that in the proof of Theorem 1, $f\left(\delta_{S}^{\star}\right)>0$ holds for every $\delta_{S}^{\star} \in(0, \pi / 2)$. Hence, (48) and $\sin \left(\delta_{S}^{\star}-\delta_{B}\left(\delta_{S}^{\star}\right)\right)>0$ imply

$$
\begin{aligned}
r_{S} v_{\infty} \sin \delta_{S}^{\star} \sqrt{f\left(\delta_{S}^{\star}\right)-r_{S}^{2} r_{L}^{2} i_{P}^{2}} & \\
& >r_{S} r_{L} i_{P}\left(r_{L} v_{S}+r_{S} v_{\infty} \cos \delta_{S}^{\star}\right)
\end{aligned}
$$

For $a_{21}\left(\delta_{S}^{\star}\right)$ in (33), (49) yields

$$
\frac{r_{S}^{2} r_{L}^{2} v_{S} v_{\infty} i_{P} \sin \delta_{S}^{\star}}{\sqrt{f\left(\delta_{S}^{\star}\right)-r_{S}^{2} r_{L}^{2} i_{P}^{2}}}=\frac{r_{S}^{3} r_{L}^{2} v_{S} v_{\infty}^{2} i_{P} \sin ^{2} \delta_{S}^{\star}}{r_{S} v_{\infty} \sin \delta_{S}^{\star} \sqrt{f\left(\delta_{S}^{\star}\right)-r_{S}^{2} r_{L}^{2} i_{P}^{2}}}
$$




$$
\begin{aligned}
& <\frac{r_{S}^{3} r_{L}^{2} v_{S} v_{\infty}^{2} i_{P} \sin ^{2} \delta_{S}^{\star}}{r_{S} r_{L} i_{P}\left(r_{L} v_{S}+r_{S} v_{\infty} \cos \delta_{S}^{\star}\right)} \\
& =\frac{r_{S}^{2} r_{L} v_{S} v_{\infty}^{2} \sin ^{2} \delta_{S}^{\star}}{r_{L} v_{S}+r_{S} v_{\infty} \cos \delta_{S}^{\star}} .
\end{aligned}
$$

In addition, we obtain

$$
\begin{aligned}
& \frac{1}{f\left(\delta_{S}^{\star}\right)}\left(\frac{r_{S}^{2} r_{L}^{2} v_{S} v_{\infty} i_{P} \sin \delta_{S}^{\star}}{\sqrt{f\left(\delta_{S}^{\star}\right)-r_{S}^{2} r_{L}^{2} i_{P}^{2}}}+r_{L}^{2} v_{S}^{2}+r_{S} r_{L} v_{S} v_{\infty} \cos \delta_{S}^{\star}\right) \\
& <\frac{1}{f\left(\delta_{S}^{\star}\right)}\left(\frac{r_{S}^{2} r_{L} v_{S} v_{\infty}^{2} \sin ^{2} \delta_{S}^{\star}}{r_{L} v_{S}+r_{S} v_{\infty} \cos \delta_{S}^{\star}}+r_{L}^{2} v_{S}^{2}+r_{S} r_{L} v_{S} v_{\infty} \cos \delta_{S}^{\star}\right) \\
& =\frac{1}{f\left(\delta_{S}^{\star}\right)}\left(\frac{r_{L} v_{S}\left(r_{S}^{2} v_{\infty}^{2} \sin ^{2} \delta_{S}^{\star}+\left(r_{L} v_{S}+r_{S} v_{\infty} \cos \delta_{S}^{\star}\right)^{2}\right)}{r_{L} v_{S}+r_{S} v_{\infty} \cos \delta_{S}^{\star}}\right) \\
& =\frac{r_{L} v_{S}}{r_{L} v_{S}+r_{S} v_{\infty} \cos \delta_{S}^{\star}} \\
& <1
\end{aligned}
$$

where the first inequality is derived by (50) and $f\left(\delta_{S}^{\star}\right)>0$ for every $\delta_{S}^{\star} \in(0, \pi / 2)$, the second and third equalities are given by a simple calculation and (22), respectively, and the last inequality follows from $\cos \delta_{S}^{\star}>0$ for every $\delta_{S}^{\star} \in(0, \pi / 2)$. This, together with (33), $\cos \delta_{S}^{\star}>0$, and $\sin \left(\delta_{S}^{\star}-\delta_{B}\left(\delta_{S}^{\star}\right)\right)>$ 0 , shows $a_{21}\left(\delta_{S}^{\star}\right)<0$.

\section{C.2 Case of $\sin \left(\delta_{s}^{\star}-\delta_{B}\left(\delta_{s}^{\star}\right)\right)<0$}

In a similar way to that in the previous section, we have

$$
\frac{r_{S}^{2} r_{L}^{2} v_{S} v_{\infty} i_{P} \sin \delta_{S}^{\star}}{\sqrt{f\left(\delta_{S}^{\star}\right)-r_{S}^{2} r_{L}^{2} i_{P}^{2}}}>\frac{r_{S}^{2} r_{L} v_{S} v_{\infty}^{2} \sin ^{2} \delta_{S}^{\star}}{r_{L} v_{S}+r_{S} v_{\infty} \cos \delta_{S}^{\star}}
$$

instead of (50). Thus, similar to (51), we obtain

$$
\begin{aligned}
& \frac{1}{f\left(\delta_{S}^{\star}\right)}\left(\frac{r_{S}^{2} r_{L}^{2} v_{S} v_{\infty} i_{P} \sin \delta_{S}^{\star}}{\sqrt{f\left(\delta_{S}^{\star}\right)-r_{S}^{2} r_{L}^{2} i_{P}^{2}}}+r_{L}^{2} v_{S}^{2}+r_{S} r_{L} v_{S} v_{\infty} \cos \delta_{S}^{\star}\right) \\
& >\frac{r_{L} v_{S}}{r_{L} v_{S}+r_{S} v_{\infty} \cos \delta_{S}^{\star}} .
\end{aligned}
$$

This and (33) provide

$$
\begin{aligned}
a_{21}\left(\delta_{S}^{\star}\right)< & -\frac{v_{S} v_{\infty} \cos \delta_{S}^{\star}}{M\left(r_{S}+r_{L}\right)}-\frac{r_{L} v_{S} i_{P} \sin \left(\delta_{S}^{\star}-\delta_{B}\left(\delta_{S}^{\star}\right)\right)}{M\left(r_{S}+r_{L}\right)} \\
& \times\left(1-\frac{r_{L} v_{S}}{r_{L} v_{S}+r_{S} v_{\infty} \cos \delta_{S}^{\star}}\right) \\
= & -\frac{1}{M\left(r_{S}+r_{L}\right)}\left(v_{S} v_{\infty} \cos \delta_{S}^{\star}+r_{L} v_{S} i_{P}\right.
\end{aligned}
$$

$$
\begin{gathered}
\times\left(-\frac{r_{S} r_{L} i_{P}\left(r_{L} v_{S}+r_{S} v_{\infty} \cos \delta_{S}^{\star}\right)}{f\left(\delta_{S}^{\star}\right)}\right. \\
\left.+\frac{r_{S} v_{\infty} \sin \delta_{S}^{\star} \sqrt{f\left(\delta_{S}^{\star}\right)-r_{S}^{2} r_{L}^{2} i_{P}^{2}}}{f\left(\delta_{S}^{\star}\right)}\right)
\end{gathered}
$$

$$
\begin{aligned}
& \left.\times\left(1-\frac{r_{L} v_{S}}{r_{L} v_{S}+r_{S} v_{\infty} \cos \delta_{S}^{\star}}\right)\right) \\
= & -\frac{1}{M\left(r_{S}+r_{L}\right)}\left(v_{S} v_{\infty} \cos \delta_{S}^{\star}-\frac{r_{S}^{2} r_{L}^{2} v_{S} v_{\infty} i_{P}^{2} \cos \delta_{S}^{\star}}{f\left(\delta_{S}^{\star}\right)}\right. \\
+ & \left.\frac{r_{S}^{2} r_{L} v_{S} v_{\infty} i_{P} \sin \delta_{S}^{\star} \cos \delta_{S}^{\star} \sqrt{f\left(\delta_{S}^{\star}\right)-r_{S}^{2} r_{L}^{2} i_{P}^{2}}}{f\left(\delta_{S}^{\star}\right)\left(r_{L} v_{S}+r_{S} v_{\infty} \cos \delta_{S}^{\star}\right)}\right),
\end{aligned}
$$

where the first inequality is obtained from (53) and $\sin \left(\delta_{S}^{\star}-\right.$ $\left.\delta_{B}\left(\delta_{S}^{\star}\right)\right)<0$, the second equality follows from (48), and the last one is given by a simple calculation. Thus, we obtain

$$
\begin{gathered}
a_{21}\left(\delta_{S}^{\star}\right)<-\frac{1}{M\left(r_{S}+r_{L}\right)}\left(v_{S} v_{\infty} \cos \delta_{S}^{\star}\right. \\
-\frac{\left(r_{L} v_{S}-r_{S} v_{\infty}\right)^{2} v_{S} v_{\infty} \cos \delta_{S}^{\star}}{f\left(\delta_{S}^{\star}\right)} \\
\left.+\frac{r_{S}^{2} r_{L} v_{S} v_{\infty} i_{P} \sin \delta_{S}^{\star} \cos \delta_{S}^{\star} \sqrt{f\left(\delta_{S}^{\star}\right)-r_{S}^{2} r_{L}^{2} i_{P}^{2}}}{f\left(\delta_{S}^{\star}\right)\left(r_{L} v_{S}+r_{S} v_{\infty} \cos \delta_{S}^{\star}\right)}\right) \\
=\frac{1}{M\left(r_{S}+r_{L}\right)}\left(\frac{2 r_{S} r_{L} v_{S}^{2} v_{\infty}^{2} \cos \delta_{S}^{\star}\left(\cos \delta_{S}^{\star}+1\right)}{f\left(\delta_{S}^{\star}\right)}\right) \\
\left.+\frac{r_{S}^{2} r_{L} v_{S} v_{\infty} i_{P} \sin \delta_{S}^{\star} \cos \delta_{S}^{\star} \sqrt{f\left(\delta_{S}^{\star}\right)-r_{S}^{2} r_{L}^{2} i_{P}^{2}}}{f\left(\delta_{S}^{\star}\right)\left(r_{L} v_{S}+r_{S} v_{\infty} \cos \delta_{S}^{\star}\right)}\right) \\
<0, \quad+5
\end{gathered}
$$

where the first inequality is derived by (11) and $f\left(\delta_{S}^{\star}\right)>0$ for every $\delta_{S}^{\star} \in(0, \pi / 2)$, the second equality is given by (22), and the last inequality follows from $\sin \delta_{S}^{\star}>0, \cos \delta_{S}^{\star}>0$, and $f\left(\delta_{S}^{\star}\right)>0$ for every $\delta_{S}^{\star} \in(0, \pi / 2)$. From (55), the proof is completed.

\section{C.3 Case of $\sin \left(\delta_{s}^{\star}-\delta_{B}\left(\delta_{S}^{\star}\right)\right)=0$}

As a direct consequence of (33) and $\cos \delta_{S}^{\star}>0$ for every $\delta_{S}^{\star} \in(0, \pi / 2), a_{21}\left(\delta_{S}^{\star}\right)<0$ is established. 


\section{References}

1. Aly MM, Abdel-Akher M, Ziadi Z, Senjyu T (2014) Assessment of reactive power contribution of photovoltaic energy systems on voltage profile and stability of distribution systems. Int J Electr Power Energy Syst 61:665-672

2. Du W, Wang H, Xiao LY (2012) Power system small-signal stability as affected by grid-connected photovoltaic generation. Eur Trans Electr Power 22(5):688-703

3. Eftekharnejad S, Vittal V, Heydt GT, Keel B, Loehr J (2013) Small signal stability assessment of power systems with increased penetration of photovoltaic generation: a case study. IEEE Trans Sustain Energy 4(4):960-967

4. Izumi S, Karakawa Y, Xin X, Yamasaki T (2016) Stability analysis of power systems with photovoltaic generators. In: Proceedings of the 55th IEEE conference on decision and control, pp 4136-4141

5. Krommydas KF, Alexandridis AT (2014) Nonlinear analysis of a grid-connected photovoltaic/dc-load system driven by local current-mode controllers. In: Proceedings of the 53rd IEEE conference on decision and control, pp 3909-3914

6. Kundur P (1994) Power system stability and control. McGraw-Hill, New York

7. Li Y, Ishikawa M (2017) Statistical analysis of power system sensitivity under random penetration of photovoltaic generation. Asian J Control 19(5):1688-1698

8. Liu S, Liu PX, Wang X (2016) Stochastic small-signal stability analysis of grid-connected photovoltaic systems. IEEE Trans Indust Electr 63(2):1027-1038

9. Rezkallah M, Sharma SK, Chandra A, Singh B, Rousse DR (2017) Lyapunov function and sliding mode control approach for the solarPV grid interface system. IEEE Trans Indust Electron 64(1):785795

10. Sakamoto N, Taniguchi H, Ota Y, Nakajima T, Chinuki T (2012) Transient stability study of one-machine-to-infinite-bus power system under large penetration of PV generation. IEEJ Trans Power Energy 132(1):9-15 (in Japanese)
11. Shah R, Mithulananthan N, Bansal RC, Ramachandaramurthy VK (2015) A review of key power system stability challenges for largescale PV integration. Renew Sustain Energy Rev 41:1423-1436

12. Tan YT, Kirschen DS, Jenkins N (2004) A model of PV generation suitable for stability analysis. IEEE Trans Energy Convers 19(4):748-755

13. Xue Y, Manjrekar M, Lin C, Tamayo M, Jiang JN (2011) Voltage stability and sensitivity analysis of grid-connected photovoltaic systems. In: Proceedings of the 2011 IEEE power and energy society general meeting, pp 1-7

14. Yagami M, Ishikawa S, Ichinohe Y, Misawa K, Tamura J (2014) Transient stability analysis of power system with photovoltaic systems installed. In: Proceedings of the 3rd renewable power generation conference, pp 1-6

15. Yahya A, El Fadil H, Oulcaid M, Ammeh L, Giri F, Guerrero JM (2018) Control of grid connected photovoltaic systems with microinverters: new theoretical design and numerical evaluation. Asian J Control 20(2):906-918

16. Yan R, Saha TK (2012) Investigation of voltage stability for residential customers due to high photovoltaic penetrations. IEEE Trans Power Syst 27(2):651-662

17. Zhou Y, Li Y, Liu W, Yu D, Li Z, Liu J (2017) The stochastic response surface method for small-signal stability study of power system with probabilistic uncertainties in correlated photovoltaic and loads. IEEE Trans Power Syst 32(6):4551-4559

18. Zwillinger D (2002) CRC standard mathematical tables and formulae, 31st edn. CRC Press, Boca Raton

Publisher's Note Springer Nature remains neutral with regard to jurisdictional claims in published maps and institutional affiliations. 